\title{
Thalamocortical Sensorimotor Circuit in Multiple Sclerosis: An Integrated Structural and Electrophysiological Assessment
}

\author{
Maria Luisa Dell'Acqua,' Doriana Landi,' Giancarlo Zito, ${ }^{2,3}$ Filippo \\ Zappasodi, ${ }^{4}$ Domenico Lupoi, ${ }^{2}$ Paolo M. Rossini, ${ }^{1,2,3}$ Maria M. Filippi, ${ }^{2}$ \\ and Franca Tecchio ${ }^{5,3 *}$ \\ ${ }^{1}$ Neurologia Clinica, Universitá Campus Bio-Medico, Roma, Italy \\ ${ }^{2}$ Dipartimento di Neuroscienze ed Unità Operativa Complessa Radiologia Diagnostica ed \\ Interventistica, AFaR, Ospedale Fatebenefratelli, Isola Tiberina, Roma, Italy \\ ${ }^{3}$ Casa di Cura San Raffaele Cassino e IRCCS San Raffaele Pisana, Italy \\ ${ }^{4}$ Dipartimento di Scienze Cliniche e delle Bioimmagini, Università "G.D'Annunzio," Chieti, Italy \\ ${ }^{5}$ ISTC-CNR, Unità MEG-Ospedale Fatebenefratelli, Isola Tiberina, Roma, Italy
}

\begin{abstract}
Demyelination and axonal damage are pathologic hallmarks of multiple sclerosis (MS), leading to loss of neuronal synchronization, functional disconnection amongst brain relays, and clinical sequelae. To investigate these properties, the primary component of the sensorimotor network was analyzed in mildly disabled Relapsing-Remitting MS patients without sensory symptoms at the time of the investigation. By magnetoencephalography (MEG), the recruitment pattern within the primary sensory (S1) and motor (M1) areas was estimated through the morphology of the early components of somatosensory evoked magnetic fields (SEFs), after evaluating the S1 responsiveness to sensory inputs from the contralateral arm. In each hemisphere, network recruitment properties were correlated with ispilateral thalamus volume, estimated by morphometric techniques upon high-resolution 3D structural magnetic resonance images (MRI). S1 activation was preserved, whereas SEF morphology was strikingly distorted in MS patients, marking a disruption of primary somatosensory network patterning. An unbalance of S1-M1 dynamic recruitment was documented and correlated with the thalamic volume reduction in the left hemisphere. These findings support the model of MS as a disconnection syndrome, with major susceptibility to damage experienced by nodes belonging to more frequently recruited and highly specialized networks. Hum Brain Mapp 31:1588-1600, 2010. @ 2010 Wiley-Liss, Inc.
\end{abstract}

Key words: magnetoencephalography; magnetic resonance imaging; functional connectivity; interhemispheric asymmetry; thalamus volume

Contract grant sponsor: European Community's Seventh Framework Programme (project MEGMRI); Contract grant number: 200859; Contract grant sponsor: FISM-Fondazione Italiana Sclerosi Multipla ("FaMuS" project); Contract grant number: Cod.2009/R/23.

*Correspondence to: Franca Tecchio, ISTC-CNR, Unità MEG, Ospedale Fatebenefratelli Isola Tiberina, 00186 Rome, Italy.
E-mail: franca.tecchio@istc.cnr.it

Received for publication 24 September 2009; Accepted 2 November 2009

DOI: $10.1002 / \mathrm{hbm} .20961$

Published online 16 February 2010 in Wiley Online Library (wileyonlinelibrary.com). 


\section{INTRODUCTION}

Demyelination and axonal damage are considered pathological hallmarks of multiple sclerosis (MS) since the first reports about this disease [Charcot, 1868]. By impairing the conduction of neural signals, they alter mechanisms of neural firing synchronization affecting the connectivity between subcortical and spinal targets and cortico-cortical pathways. A disconnection in large-scale cortical networks is an expected and well-known effect of the disease, possibly subtending the neuropsychological deficits encountered throughout its natural history. In this respect, MS has been defined as a "multiple disconnection syndrome" [Calabrese, 2006; He et al., in press]. Furthermore, an abnormal pattern of small-range functional connectivity among regions involved in sensorimotor control has been evidenced as a further early feature of the disease, both in resting state [De Luca et al., 2005; Lowe et al., 2002] and during the execution of simple movements [Casadio et al., 2008; Rocca et al., 2007, 2008].

The sensorimotor network imbalance could be a consequence of the damage to both its relays and its interconnecting white-matter bundles. Each relay is functionally organized to give its unique support to the behavioral pattern of the ultimate network and may be engaged in a number of different domains of behavior subserved by anatomically distinct networks. The wide clinical spectrum of disconnection syndromes is mainly dependent on which node or connecting axons are impaired [Schmahmann and Pandya, 2008].

In MS, a strong effort is being devoted to better elucidate connectivity impairments either from an anatomical [Audoin et al., 2007; Cader et al., 2007; Cercignani et al., 2002; Reich et al., 2007] and/or functional perspective [Au Duong et al., 2005; Audoin et al., 2006; Cader et al., 2006; Horwitz, 2003; Leocani and Comi, 1999; Reddy et al., 2000].

Each sensory modality implies the recruitment of specific nervous pathways characterized by intimate timevarying spatial properties that contribute to delineate the morphology of the electrophysiological response, as detected either by electro- or magneto-encephalography. In other words, such a morphology is modality-dependent, indicating whether the delivered stimulus is auditory, visual, or somatosensory [Creutzfeldt, 1977; Desmedt and Ozaki, 1991; Mauguiere et al., 1997; Mima et al., 1996; Restuccia et al., 1999; Valeriani et al., 1998]. Even within primary somatosensory cortex (S1), the morphology of the somatosensory evoked field (SEF) differentiates the cortical structural subcomponents generating the signal, as revealed by the different response shape after stimulation of ulnar or median nerve [Tecchio et al., 2005].

A dynamical process involving interacting excitatory and inhibitory structures shapes up the morphology of SEF. As the thalamic input impinges on S1, excitatory postsynaptic potentials (EPSP) on pyramidal cells from Brodmann area (BA) 3b is reflected by the M20 SEF com- ponent detected by magnetoencephalography (MEG) [Hari et al., 1984; Kawamura et al., 1996; Mauguiere et al., 1997; Rossini et al., 1994]. In the following 10s of milliseconds, an inhibitory postsynaptic potential (IPSP) induced by the local gabaergic stellate cells on pyramidal neurons of area $3 \mathrm{~b}$ and an EPSP on pyramidal cells of primary motor cortex (M1, BA 4) are concurrently generated, resulting in the M30 SEF component [Huang et al., 2000; Kawamura et al., 1996; Niimi et al., 2008; Tecchio et al., 2005; see Fig. 1].

Although the M30 component represents the peak of activation of these S1-M1 neuronal pools, the morphology of the evoked response reflects the dynamics of their balancing engagement along the whole recruitment period. Because functional connectivity can be defined as timevarying aggregation of two or more neuronal pools into a network sustaining a given cerebral process, SEF morphology reflects the functional connectivity within the primary sensorimotor areas. A quantitative assessment of the SEF morphology had been previously obtained [Morf_S1M1, Tecchio et al., 2000, 2005; Fig. 2], focusing the analysis on the 20-ms interval following the arrival of sensory input to the primary sensory cortex (M20). Information upon SEF shape as a marker of the spatiotemporal recruitment pattern is derived normalizing for the signal amplitude, thus describing connectivity properties independently of the amount of synchronously recruited neurons. Thalamus is a key subcortical node providing the whole projection contingent to S1; several studies covering pathology [Brownell and Hughes, 1962; Cifelli et al., 2002] and magnetic resonance images (MRI) issues [Cifelli et al., 2002; Fabiano et al., 2003; Inglese et al., 2004, 2007; Ormerod et al., 1987; Wylezinska et al., 2003] demonstrate its involvement in MS. Recent voxel-based morphometry studies demonstrated thalamic gray matter (GM) loss in patients with primary progressive, early RR-MS [Houtchens et al., 2007], and pediatric MS [Audoin et al., 2006; Mesaros et al., 2008; Sepulcre et al., 2006]; a strict correlation between thalamic GM loss and T2 lesion burden was also found.

In this study, the functional pattern of connectivity within S1 and M1 was investigated, during the processing of a simple sensory stimulus in a cohort of MS patients. Potential effects of thalamic volumetry changes upon the network recruitment properties were also assessed.

\section{SUBJECTS AND METHODS}

\section{Patients}

To test the sensitivity of our analysis, we purposefully selected patients with no or very mild disability, in remitting phase and without complaints of sensory deficits at the time of evaluation. Patients were recruited on the basis of the following criteria: diagnosis of MS according to McDonalds Criteria [2001]; Relapsing-Remitting (RR) clinical course [Lublin and Reingold, 1996]; absence of clinical or radiological relapse for at least 3 months preceding the 
M20 ECD

EPSP on S1 pyramidal cells

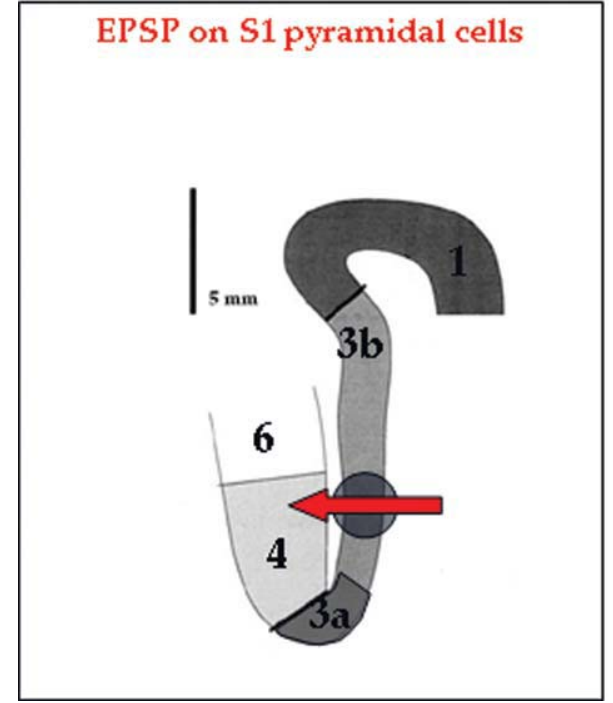

M30 ECD

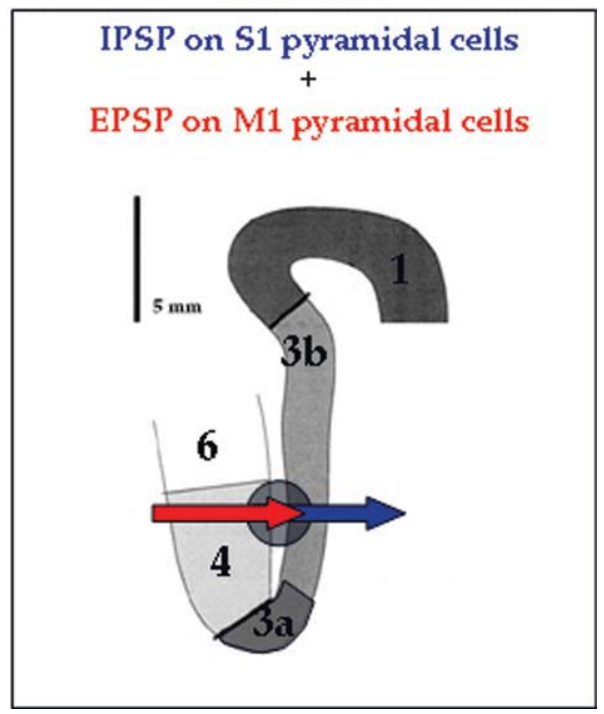

Figure I.

Cortical generators of SEF M20 and M30 components. Sagittal section of primary sensory and motor areas with schematic representation of currents subtending $M 20$ and $M 30$ generators. Red row indicates current induced by EPSP and blue row the effect of IPSP. Black circle represent the position of the equivalent current dipole (ECD) of each component. Left: the M20 component is mainly generated by EPSP impinging on pyramidal neurons in primary somatosensory BA $3 \mathrm{~b}$. Right: the currents

associated with the IPSPs impinging on pyramidal neurons in $3 \mathrm{~b}$ area and the EPSPs onto BA 4 pyramidal neurons contribute in the same direction to the current, and consequently to the magnetic signal, and their sum reaches its maximum in about $10 \mathrm{~ms}$ from M20 latency, giving rise to M30 component. [Color figure can be viewed in the online issue, which is available at wileyonlinelibrary.com.]

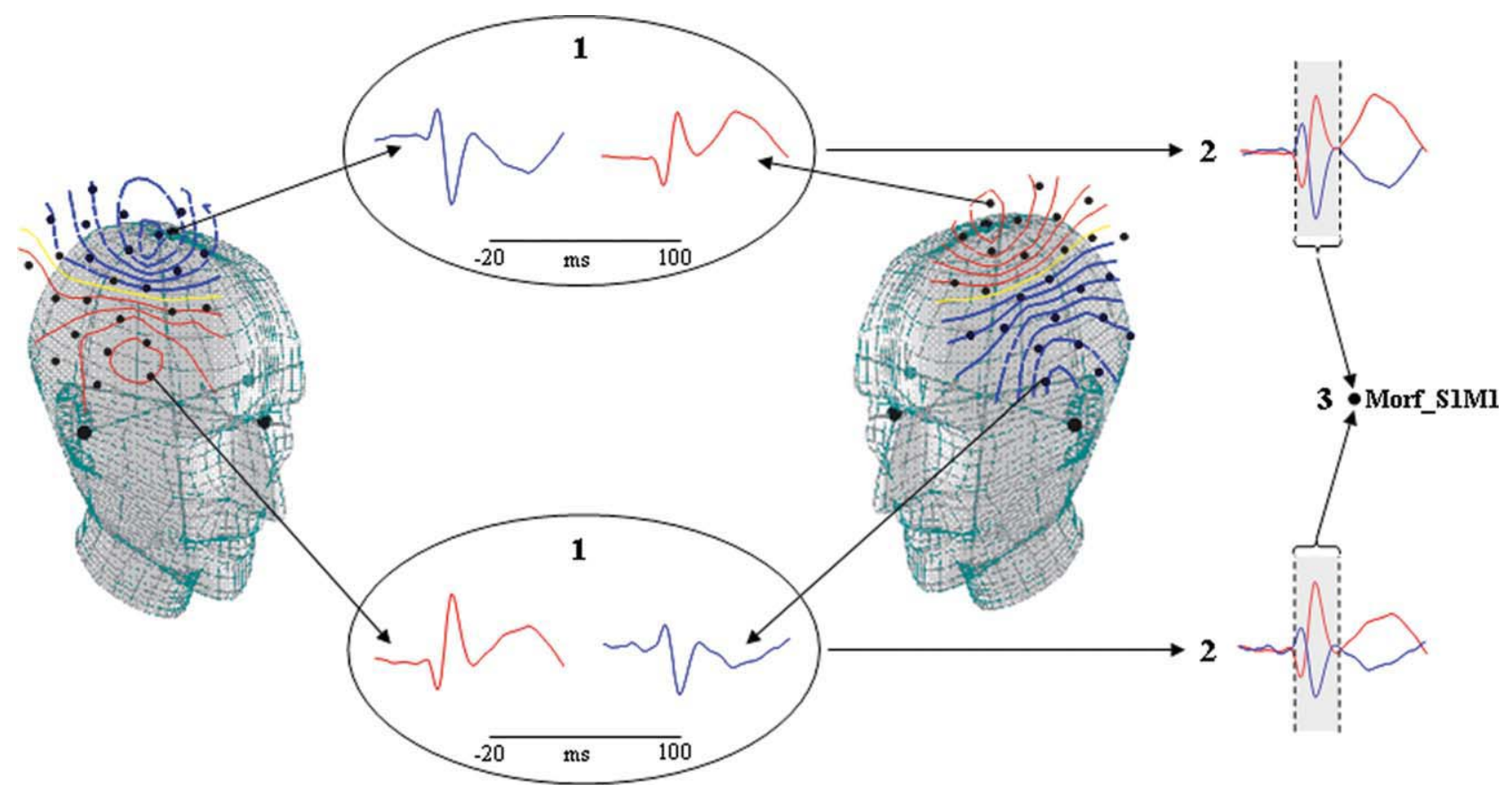

Figure 2.

Morf_SMI index definition. Steps I, 2, and 3 for the similarity index calculation (see methods section), comparing SEFs by left and right median nerve stimulation (morf_SMI). [Color figure can be viewed in the online issue, which is available at wileyonlinelibrary.com.] 
- Thalamocortical Sensorimotor Circuit in RR-MS •

TABLE I. Clinical and radiological characteristics of patients

\begin{tabular}{|c|c|c|}
\hline & MS patients & Healthy controls \\
\hline Mean age, years $(S D)$ & $39(9)$ & $38(12)$ \\
\hline $\operatorname{Sex}(M: F)$ & 5:16 & $5: 16$ \\
\hline Median EDSS [5-95 percentiles] & $1.5[0-3.5]$ & \\
\hline Mean disease duration, years $(S D)$ & $10(7)$ & \\
\hline Median relapse rate [5-95 percentiles] & $0.33[0.08-2.23]$ & \\
\hline Interferon- $\beta-1 a$, number of patients & 10 & \\
\hline Median LrF, ml [5-95 percentiles] & $0.047[0.001-0.208]$ & \\
\hline Median BPF, \% $(S D)$ & $0.797(0.045)$ & $0.849(0.049)$ \\
\hline Left thalamus volume, ml (SD) & $6.84(0.83)$ & $7.28(0.37)$ \\
\hline Right thalamus volume, $\mathrm{ml}(S D)$ & $6.56(0.55)$ & $6.81(0.44)$ \\
\hline
\end{tabular}

Extended Disability Scale Score (EDSS), Lesion relative Fraction (LrF), and Brain Parenchyma Factor (BPF). Values in bold differ between patients and controls. Left thalamic volumes were larger than right [Ahsan et al., 2007].

study; low degree of disability as estimated by an Extended Disability Scale Score $\leq 3.5$ [Kurtzke, 1983]; absence of upper limb positive or negative sensory symptoms on neurological examination at the time of the study; no treatment with corticosteroids or psychotropic drugs within the previous 3 months.

Ethic Committee of "S. Giovanni Calibita" Fatebenefratelli Hospital approved the study, and all patients gave their written consent, according to the Helsinki Declaration.

Twenty-one (age range 26-60, mean $39.2 \pm 9.2$ years; 5 males and 16 females) right-handed (Edinburgh Inventory $>$ 85; [Oldfield, 1971]) patients and 21 healthy subjects (age range 24-60, mean $38.4 \pm 11.7$ years; 5 males and 16 females) were recruited (Table I). Patient and control group ages did not differ (independent sample $t$-test; $P=$ 0.804, applied after checking that the distributions did not differ from a Gaussian, Kolmogorov-Smirnov $P>0.600$ for both).

\section{MEG Study}

\section{Recordings}

Measurements were carried out using a 28-channel system (16 internal axial gradiometers and 9 peripheral magnetometers, 3 balancing magnetometer being devoted to noise reduction) covering a total area of about $180 \mathrm{~cm}^{2}$, operating in a magnetically shielded room (Vacuumschmelze GMBH) [Tecchio et al., 1997]. Subjects comfortably laid on a nonmagnetic hospital bed and were asked to keep their eyes open to reduce the effects of rest occipital activity. The magnetic field distribution over the scalp was recorded after separate electrical stimulation of the median nerve at the wrist of left and right hand, via a single position on the hemisphere contralateral to the stimulated side, by centering the recording apparatus on the C3/C4 positions of the 10-20 International EEG system. Galvanic stimuli via surface disks were delivered with standard characteristics, that is, $0.2-\mathrm{ms}$ pulses at about 2
$\mathrm{Hz}$ of repetition rate and stimulus intensity adjusted to elicit a painless thumb twitch. No specific attention was required attending the stimuli. Data were bandpass filtered $(0.48-250 \mathrm{~Hz})$ and sampled at $1 \mathrm{KHz}$.

The whole magnetoencephalography (MEG) analysis was performed via in-house developed software in $\mathrm{C}++$ (for review, see Del Gratta et al. [2001] and Romani and Pizzella [1990]). A semiautomatic ICA-based procedure [Barbati et al., 2004] was applied off line to identify cardiac and/or ocular artefacts and remove them from contaminated trials, without any need to exclude the cerebral signal in those periods from further analysis. Saturated epochs were rejected by visual inspection. To obtain SEF, about 300 artifact-free trials were averaged on $[-20,100]$ $\mathrm{ms}$ time epoch, 0 being the moment of stimulus delivery to the median nerve at wrist. Amplitudes of SEFs were calculated with respect to a baseline level chosen as the mean value of the 5-15-ms poststimulus epoch. The latencies of the earliest SEF components M20 and M30 were identified as the amplitude peaks in the evoked responses at about 20 and $30 \mathrm{~ms}$ from the stimulus occurrence.

\section{Shape evaluation of SEFs}

To quantitatively estimate the SEF morphology, we used the index Morf_SM1 published in Tecchio et al. [2000] (see Fig. 2). Morf_SM1 is calculated in the time window including exclusively the M20 and M30 components, generated in primary sensory and motor cortical areas. Left and right M20 onsets are aligned each other allowing the comparison of SEF morphology, independently of possible differences in M20 latency between the two hemispheres.

In details, Morf_SM1 was obtained as follows (step numbers and labels accordingly to Fig. 2)

1. The two channels showing opposite polarity and maximal amplitude in each hemisphere were selected;

2. The channels in the two hemispheres were aligned to the M20 onset and the correlation coefficients of each channel with the one in the corresponding cerebral 
region of the other hemisphere were calculated, in the time period lasting 20 ms from the M20 onset;

3. These two correlation indexes were averaged.

Thus, the calculus is:

$\operatorname{sim}=-\frac{1}{2}\left[\left(\frac{\sum_{i=1}^{20} x_{i} y_{i}}{\sqrt{\sum_{i=1}^{20} x_{i}^{2} \sum_{i=1}^{20} y_{i}^{2}}}\right)_{\text {vertex }}+\left(\frac{\sum_{i=1}^{20} x_{i} y_{i}}{\sqrt{\sum_{i=1}^{20} x_{i}^{2} \sum_{i=1}^{20} y_{i}^{2}}}\right)_{\text {temp.front }}\right]$

where $x$ and $y$ are the four selected channels, each pair ( $x$, $y$ ) in the corresponding cerebral region more proximal to the vertex (vertex) and in the temporo-frontal (temp.front) areas, respectively. To have a positive similarity index between 0 (no similarity) and 1 (perfect shape superimposition), the average was multiplied by minus one, because the channels in the two corresponding cerebral regions (vertex and temporo-frontal) of left and right hemispheres have opposite polarities.

The analysis of the channels giving the minimum/maximum responses at the latency of the first component is motivated by the need to catch the best and earliest activation of the S1. Indeed, the first component around $20 \mathrm{~ms}$ is accepted as the marker of the signal arrival to S1; moreover, at this peak latency, a consistent dipolar distribution is generated, and the minimum/maximum channels are the best descriptors of activation. A time window lasting $20 \mathrm{~ms}$ is chosen to focus the analysis on the activation properties of neural network within the primary sensory and motor areas: in fact, this time period includes the first two response components (M20 and M30), mainly generated in these cortical regions. To be noted, M20 and M30 sources are very near each other and the minimum/maximum channels defined on M20 are well representative for M30 component too.

\section{Recruitment pattern of $\mathrm{M} 20$ and $\mathrm{M} 30$ generators}

As an estimate of the number of the recruited neuronal pools and their location, we considered the strength and position of the M20 and M30 components' generators. The equivalent current dipole (ECD) inside a homogeneously conducting sphere was used for the solution of the inverse problem [Del Gratta et al., 2001; Ilmoniemi, 1993]. The localization results were accepted only if their explained variance $(\mathrm{ExV})$ was above $90 \%$. Time points corresponding to maximal field power around the two earliest components were considered as latencies of M20 and M30 waves. ECD positions at these latencies (only if ExV > 90\%) were expressed in a coordinate system defined, so that central axis passed through the midline between the two hemispheres: $x$ - and $y$-axis passing through the mean point between the two preauricular points, $y$-axis outgoing from nasion, positive $x$-axis rightward on the plane including nasion and preauricular points, thus positive $z$-axis upward directed. In this way, equal $\times$ absolute values in the two hemispheres corresponded to symmetric positions in respect with the interhemispheric fissure. This property was exploited to estimate the interhemispheric "asymmetry" of left and right ECD positions. In detail, the position of M20 ECD in each hemisphere was calculated; the left position was projected onto the right (considering the absolute value of left ECD $x$-coordinate); finally, the Euclidean distance between these two ECDs was obtained. We applied the same procedure to M30 ECDs. Moreover, the Euclidean distance between M30 and M20 ECDs (locM30-M20) was calculated in each hemisphere to assess their reciprocal displacement. In fact, a reduction of Morf_S1M1 can be due to modifications in timing, number, and position of neuronal elements' firing generating M20 and M30 components. Specifically, a reduction of Morf_S1M1 may result from an excessive interhemispheric asymmetry of M20 or M30 ECD positions or a delay or displacement of M30 ECD with respect to the M20 ECD in a single hemisphere.

\section{MRI exam}

\section{MRI acquisition}

Imaging was performed at the Radiology Unit of the Hospital, equipped with an Achieva 1.5 T scanner (Philips Medical Systems, Best, The Netherlands), provided of 33 $\mathrm{mT} / \mathrm{m}$ gradient amplitude, online $2 \mathrm{D} / 3 \mathrm{D}$ geometric distortion correction and a standard quadrature head coil. The acquisition protocol consisted of one 3D high-res anatomical sequence and routine 2D sequences for lesion characterization. The former was empirically optimized to increase grey/white-matter image contrast and to allow reliable segmentation of subcortical structures, in particular, the thalamus (T1-weighted Turbo Field Echo TR/TE/ $\mathrm{FA}=7.0 \mathrm{~ms} / 3.16 \mathrm{~ms} / 8^{\circ} ; 256^{2}$ matrix, 160 coronal contiguous slices, in-plane resolution $1 \times 1 \mathrm{~mm}$ ). The $2 \mathrm{D}$ sequences included a Dual Turbo Spin Echo, a Fluid Attenuated Inversion Recovery (FLAIR) and two T1-Spin Echo sequences before and after intravenous injection of the contrast agent gadolinium following acquisition parameters as detailed in Tecchio et al. [2008].

\section{Lesion Characterization}

For details regarding lesion characterization, optimized segmentation and volumes calculation for normalization, see Tecchio et al. [2008]. In this study, the lesion load was estimated as the lesion relative fraction $(\mathrm{LrF})$ defined as the total lesion volume (TLV), that is, the volume of the whole T2 white-matter hyperintensities (WMHy), normalized by the total white-matter volume. Moreover, as an estimate of whole brain atrophy, the brain parenchymal factor (BPF) was considered, defined as the sum of volumes of normal-appearing white matter (NAWM), TLV, 
and GM volumes normalized by the total intracranial volume.

\section{Thalamus Volumetry}

Axial and sagittal editing was applied to 3D volumetric datasets to facilitate the neuroanatomical landmarks definition with MRICro software [Brett et al., 2001]. The landmarks used for the thalamus were the mammillary bodies as the most anterior boundary and the point of emergence of the thalamus under the crus fornix as the posterior one, bearing in mind that the ventralis anterior nucleus is dorsal to the hypothalamus, and thalamus is bounded laterally by the internal capsule, dorsally by the lateral ventricle and medially by the third ventricle. The inferior border was defined when the thalamus merged with the brain stem and the superior border, by the main body of the lateral ventricle [Duvernoy, 1991]. After entering the PACS system of the Department, the DICOM images were postprocessed on a dual processor Linux workstation. All subjects underwent an identical imaging protocol. The MRI scanner did not undergo major hardware upgrades during data collection. Thalami measurements upon anonymized images were performed twice by the same researcher, who was blind to clinical and neurophysiological characteristics, in randomized order, having $98.8 \%$ of results reproducibility.

The thalamus was manually derived as a volume of interest from the surrounding structures according to the abovementioned anatomical landmarks and working in parallel on the three spatial planes, with the coronal plane having a more relevant role, because the acquisition was made along the $Y$ axis.

\section{Statistical Analysis}

\section{M20 and $\mathrm{M} 30$ recruitment properties}

None ECD parameters (latency, position coordinates, and strength) distribution differed from the Gaussian (Kolmogorov-Smirnov test $P>0.200$ consistently). ANOVA designs for repeated measures were applied to M20 and M30 ECD latency, strength, and position with Hemisphere (Left, Right) and Component (M20, M30) as within subject factors and Group (MS patients, Healthy controls) as between-subject factor. For position, the 3D measure $(x, y, z)$ was submitted to the analysis design. Besides, the main Group factor indicating a different behavior of MS patients with respect to healthy subjects, a factor of interest was the interaction Group*Component, indicating specific behaviors of the M30 and M20 component generators in the two groups.

\section{Recruitment Dynamics Within SI and MI}

The Morf_S1M1 parameter distribution did not result in Gaussian-shaped and the following transformation allowed us to achieve the best fit (Kolmogorov-Smirnov test $P=0.998): y=-\ln (1-x)$, where $x=$ Morf_S1M1. Independent sample $t$-test was performed comparing the transformed Morf_S1M1 values between patients and controls.

\section{Brain and Thalamic Volume}

Because none of neuroradiological variable distributions differed from a Gaussian (consistently, Kolmogorov-Smirnov test, $P>0.200$ ), parametric tests were applied. To analyze thalamic volumes, an ANOVA with Hemisphere (Left, Right) as within-subject factor and Group (MS patients, Healthy controls) as between-subject factor was applied to the left and right thalamic volumes.

\section{Relationship Between Primary Somatosensory Recruitment/Dynamics, Neuroradiology, and Clinical Data}

For the electrophysiological measures resulting different among patients and controls bivariate parametric correlations with the neuroradiological measures were carried out. Nonparametric correlation was used to study the relationship between clinical scores and electrophysiological and neuroradiological parameters.

\section{RESULTS}

\section{Clinical Metrics}

Patients presented a median EDSS of 1.5, a mean disease duration of $10 \pm 7$ years, and a median relapse rate of 0.33 (Table I). Ten patients were on therapy with Interferon- $\beta$ 1 a.

None of the 21 patients presented any sensory symptom at the time of MEG evaluation. Thirteen patients had suffered from upper (11 pts) or lower (2 pts) limb sensory symptoms in the past.

\section{Neurophysiological Metrics}

\section{M20 and M30 latency}

In the patients' group, the M20 latency did not differ from healthy subjects [Group factor $F(1,39)=1.812, P=$ 0.186, Table II]. M30 latency was instead delayed in patients [Group factor $F(1,32)=6.927, P=0.013$, Table II].

Regarding the M20-M30 interpeak interval, we found a trend of a prolongation in the MS group with respect to healthy subjects [Group factor $F(1,32)=3.350, P=0.077$, Table II].

\section{M20 and M30 ECD strength}

The M20 and M30 ECD strengths behaved differently among patients and controls [Group ${ }^{*}$ Component factor $F(1$, 
- Dell'Acqua et al.

TABLE II. Primary sensorimotor area responsiveness in MS patients and controls

\begin{tabular}{|c|c|c|c|c|c|c|}
\hline & \multicolumn{2}{|c|}{ Left hemisphere } & \multicolumn{2}{|c|}{ Right hemisphere } & \multicolumn{2}{|c|}{ Interhemispheric difference } \\
\hline & MS pts & Ctr. & MS pts & Ctr. & MS pts & Ctr. \\
\hline M20 latency (ms) & $21.4(2.7)$ & $20.2(2.2)$ & $20.7(2.7)$ & $19.9(2.0)$ & $0.6(1.8)$ & $0.3(0.7)$ \\
\hline M20 strength (nA m) & $9.7(6.4)$ & $13.5(6.4)$ & $8.6(5.7)$ & $10.4(5.5)$ & $0.33(0.24)$ & $0.22(0.15)$ \\
\hline M20 position (mm) & $18.2(7.4)$ & $14.7(7.3)$ & $20.4(10.0)$ & $19.4(8.1)$ & $25.5(14.8)$ & $20.9(9.1)$ \\
\hline M30 latency (ms) & $33.6(4.5)$ & $30.0(3.2)$ & $32.6(4.5)$ & $30.2(3.7)$ & $0.7(3.8)$ & $-0.2(2.5)$ \\
\hline M30 strength (nA m) & $10.6(9.0)$ & $25.0(18.1)$ & $12.6(10.9)$ & $22.2(12.1)$ & $0.39(0.30)$ & $0.24(0.15)$ \\
\hline M30 position (mm) & $26.4(13.5)$ & $15.6(6.8)$ & $21.9(10.4)$ & $17.7(6.8)$ & $32.4(14.0)$ & $16.7(8.1)$ \\
\hline M30 vs. 20 delta latency (ms) & $12.3(3.9)$ & $10.0(3.0)$ & $11.5(3.7)$ & $10.5(2.8)$ & $0.6(3.6)$ & $-0.5(2.6)$ \\
\hline M30 vs. M20 delta position (mm) & $23.3(15.6)$ & $11.0(9.3)$ & $21.2(12.5)$ & $11.5(5.5)$ & $2.8(18.7)$ & $-0.5(9.7)$ \\
\hline & \multirow{2}{*}{\multicolumn{2}{|c|}{ Morf_S1M1 (dimensionless) }} & & & $1.49(0.93)$ & $2.76(0.86)$ \\
\hline & & & & & 0.77 & 0.94 \\
\hline
\end{tabular}

Timing (latency), intensity (strength), and spatial (position) characteristics (mean and standard deviation put between brackets) of the two cortical generators (M20 and M30 ECDs) recruited by the contralateral median nerve stimulation. Values in patients and controls are presented for the two hemispheres as well as for their interhemispheric differences. Position value refers to the Euclidean distance of the generator in each subject from the mean position in a control healthy population (see Tecchio et al. [1997]). For the position interhemispheric difference, we refer to the Euclidean distance between the two generators after mirroring the left in the right (changing the sign of the $x$-coordinate, as the Cartesian system has been defined such as the vertical plane passes through the interhemispheric fissure). M30 versus 20 delta refers to the latency difference and to the Euclidean distance between the M20 and M30 ECDs in each hemispheres. MS patient values in bold indicate statistically significant differences with controls. Below mean Morf_S1M1 values submitted to statistical analysis (see Methods section), their inverse transformations are provided for visualizing the difference in the interval $0-1$.

32) $=5.915, P=0.021]$. In fact, while the M20 ECD strength did not differ in the two groups in both hemispheres [reduced ANOVA model to M20 component: Group effect $F(1,33)=2.678, P=0.110]$, the M30 ECD strengths were bilaterally reduced in patients [Group effect $F(1,33)=8.331 ; P=0.007$, no interaction factor with Hemisphere, Table II].

\section{M20 and M30 ECD position}

The M20 and M30 ECD positions were symmetrically located in the control group. In patient group, the symmetry was preserved for M20 ECD position, whereas M30 ECDs were asymmetrically placed [Component*Group $F(1,29)=11.694, P=0.002]$. Moreover, in the MS group, the distance between the M20 and M30 generators was wider in both hemispheres than in controls [Group factor $F(1,29)=12.792, P=0.001$, no interaction factor with Hemisphere was present, Table II].

\section{SEF shape (Morf_SIMI)}

In our MS group, the SEF morphological similarity between the two hemispheres was reduced with respect to healthy controls [independent-samples $t$-test $t(39)=3.865$; $P=0.001$; Figs. 3 and 4, Table II].

Morf_S1M1 reduction did not depend on M30 ECD delayed recruitment, as it did not correlate with M30 latencies, nor the M20-M30 peak latency intervals in both hemispheres. It did not correlate with strength and position of any component in both hemispheres, nor with the distance between the positions of the M30 and M20 ECDs (consistently $P>0.200$ ).

As we mentioned earlier, Morf_S1M1 is sensitive to interhemispheric asymmetries of timing, number, and position of M20 and M30 ECDs. We then characterized the behavior of these metrics in each hemisphere compared to the other. The M20 ECD strengths were correlated in the two hemispheres both in healthy subjects (Pearson's $r=$ 0.728, $P<0.001)$ and patients $(r=0.786, P<0.001)$, whereas the M30 ECD strength strongly correlated in healthy subjects $(r=0.859, P<.001)$ and not in patients $(r$ $=0.040, P=0.869)$. Considering the M30 location, the large interhemispheric asymmetry of MS patients turned out to be principally due to M30 displacements occurring in the left hemisphere (Pearson's $r=0.684, P=0.020$ while $P>0.400$ consistently in the right hemisphere of MS and both hemispheres in controls).

\section{Neuroradiological Metrics}

\section{Thalamic volume}

An asymmetry in thalamic volumes was found, with the left thalamus larger than the right both in patients and controls [(Hemisphere factor $F(1,32)=32.805, P<0.0001)$, Table I]. In MS group, we observed a trend toward a thalamic volume reduction [Group factor $F(1,32)=2.869, P=$ 0.100 , Fig. 5] and a post hoc comparison showed a significant left thalamic volume reduction in patients with respect to controls (independent sample $t$-test $t(29.7)=$ $2.148, P=0.040$; patients volume $10 \%$ lower than controls, Table I). Only a trend for volume reduction in the right 


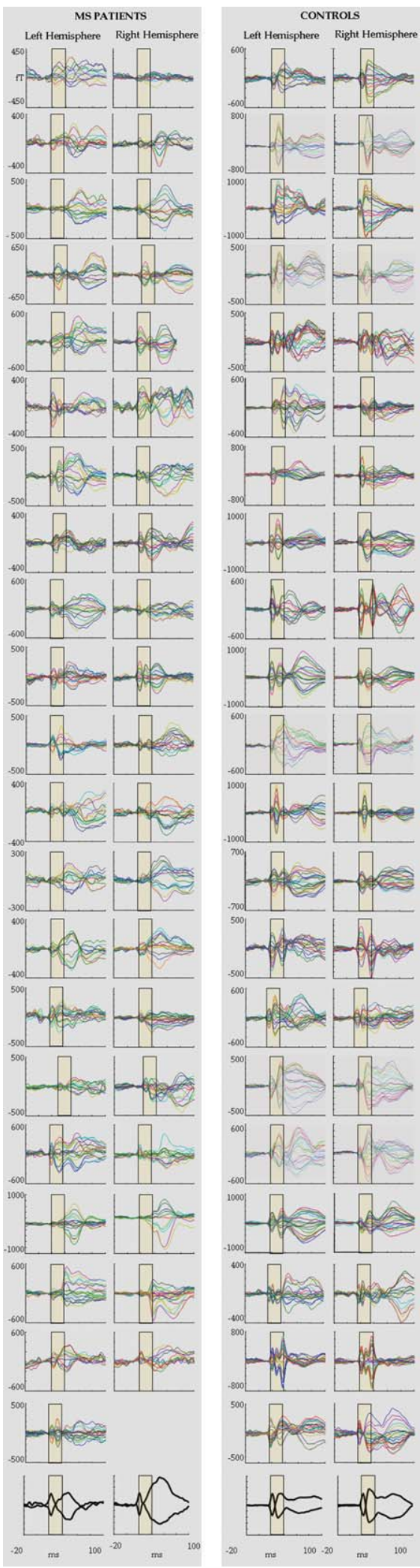

thalamus was observed $[t(32)=1.371, P=0.180]$. Because Morf_S1M1 index subtend interhemispheric asymmetries of recruitment dynamics, an index of interhemispheric asymmetry for thalamic volumes was also considered (Lt/ Rt thalamic ratio). A trend of an alteration of this ratio in patients $[t(31.1)=-1.966, P=0.058]$ was found, indicating that the left thalamic volume was more reduced than the right.

\section{Brain Parenchimal Fraction and Lesion Load}

As expected, BPF was reduced in patients with respect to controls (independent $t$-test $t(29)=2.918, P=0.007$, Table I) indicating a condition of widespread parenchimal atrophy. In our patients' group, the reduction of thalamic volume did not show a relationship with the whole brain atrophy (correlation with BPF $P>0.700$ for left and right thalamus volumes or their sum). The lesion volume calculated as LrF showed a trend toward a negative association with the thalamic volume (Pearson's $r=-0.381, P=0.088$ for the left thalamus and rho $=-0.361, P=0.108$ for the right one).

\section{Relationship Between Primary Somatosensory Recruitment/Dynamics Properties and Neuroradiology}

Initially, left and right hemispheric values were analyzed, and only the left thalamic volume correlated with

\section{Figure 3.}

SEFs traces recorded from the population of patients and normal controls. Top: In each hemisphere of both groups, superimposition of all channels in the Rolandic region averaged on stimulus arrival at the contra-lateral median nerve in the $[-20$, 100] ms period, 0 representing the stimulus delivery. On the $y$ axes, the magnetic field amplitude is represented (femtoTesla, $\mathrm{fT}$ ). The yellow boxes highlight the response time window used for Morf SIMI index estimate, whereas the entire response is shown for consistence with general literature. The morphology alteration, occurring generally in an uncorrelated manner in one or both hemispheres, disrupts the interhemispheric symmetry in MS patients. It is worth noting that while such an interhemispheric asymmetry comes out in patients, in healthy subjects, there is a striking interhemispheric similarity against a huge intersubject variability. Down left, right hemispheric data were omitted from a patient because of the presence of artifacts. Bottom: grand-average across all patients (left side) and healthy controls (right side) of the maximum and minimum channel signals (normalized to peak amplitude) in each hemisphere. It is evident the lack of structured response shape of the second cortical component in MS patients, as a consequence of distortions occurring at different times in individual patients, while in healthy subjects the two earliest components are still clearly identifiable in the mean traces. [Color figure can be viewed in the online issue, which is available at wileyonlinelibrary.com.] 


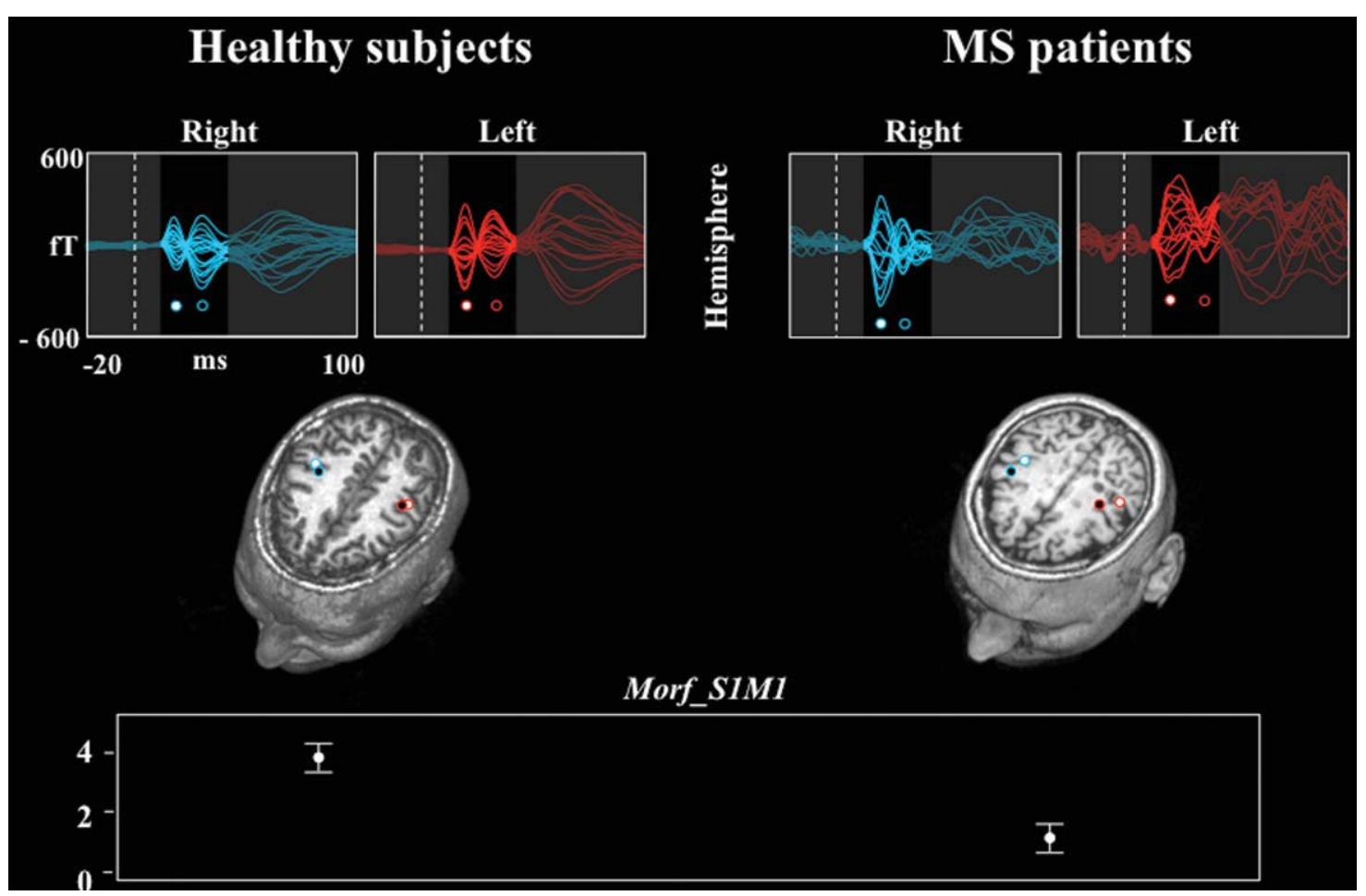

Figure 4.

SEF morphology alteration in patients. Top: the same structure as in Figure 3 of a representative healthy and RR-MS patient. A clear morphology distortion is evident in the left hemisphere of the patient, whereas M20 component is clearly identifiable in both hemispheres. Middle: in each hemisphere, the position of the ECDs explaining the cerebral activation in correspondence to the M20 (white circle) and M30 (black circle) components is

the ipsilateral position displacement of the M30 generator with respect to the M20 (Pearson's $r=-0.658, P=0.011$ ). Focusing on the relationships between the anatomical and neurophysiological interhemispheric asymmetries, the leftright thalamic ratio was positively correlated with the interhemispheric asymmetries of M30 ECD locations (Pearson's $r=0.659, P=0.020)$. No association was found with Morf_S1M1.

No electrophysiological metric correlated with the lesion burden or the whole brain atrophy $(P>0.200$ consistently).

\section{Relationship of neurophysiological and radiological features with clinical status}

Morf_S1M1 was not associated with EDSS, relapse rate, interferon- $\beta$-1a treatment and history of sensory symptoms (consistently $P>0.200$ of nonparametric correlation and independent $t$-test). Moreover, it did not depend on disease duration, even when subgrouping patients by a disease duration longer or shorter than 10 years $(P>0.200)$. A trend of positive correlation was selectively found projected onto a suitable axial slice after volumetric head reconstruction from individual MR images. Bottom: mean and standard error of the mean of the Morf_SIMI index in controls and patients, after transformation to fit its distribution to a Gaussian (see text). [Color figure can be viewed in the online issue, which is available at wileyonlinelibrary.com.]

between increasing M30 ECD displacements with respect to M20 and EDSS scores (Spearman rho $=0.581, P=0.076$ left hemisphere, rho $=0.463, P=0.082$ right). Nonparametric test in the two groups with EDSS $>2$ and $<2$ revealed statistical significant values $(P=0.041$ left and $P$ $=0.040$ right hemisphere).

Among neuroradiological variables, while thalamic volumes did not associate with the clinical state (consistently $P>200$ ), higher LrF and BPF associated with higher EDSS (Spearman rho $=0.521, P=0.015$; rho $=0.528, P=0.014$, respectively).

\section{DISCUSSION}

An integrative functional-structural study of the dynamic recruitment properties within S1 and M1 in relationship with the thalamic volume changes was conducted in a cohort of RR-MS patients.

A cortico-cortical disconnection within the primary sensorimotor cortex of our RR-MS patients was proven by a definite distortion of the morphology of the somatosensory evoked response, quantitatively indicated by the decrease 


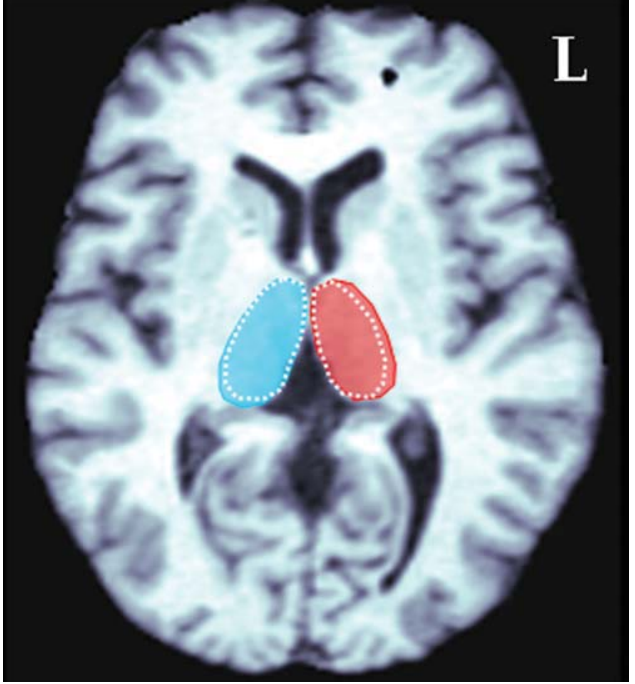

Figure 5.

Thalamus volume in MS patients and controls. Thalamic area identification in a representative normalized skull stripped (BET, www.fmrib.ox.ac.uk/fsl/bet2) axial section of a patient (F, 34 years, shown in fully coloured transparency; z-coordinate $=8$ ). The white-dotted areas indicate the average across controls of thalamic dimension on that section. The black small area in the left frontal region is part of the manually derived mask obtained in the procedure applied for lesion identification. [Color figure can be viewed in the online issue, which is available at wileyonlinelibrary.com.]

of the Morf_S1M1 index. The high symmetry of SEF shapes in the two hemispheres of the same subject, as opposite to a remarkable intersubject variability, suggests SEF morphology as an individual anatomo-functional marker of the primary somatosensory network [Tecchio et al., 2005] topology. MS profoundly impairs such an "individual marker" even in mildly disabled patients. An alteration of the response morphology can derive from the temporal dispersion among the subcompounds of the sensory volley transmission due to myelin damage. The lack of association between Morf_S1M1 and M20 or M30 ECD latency delays indicates recruitment properties also contribute-besides temporal dispersion-to morphology impairment in MS patients. The M20 ECD strength and position in patients' group were symmetric and within normative range, indicating that the ventro-posterior-lateral (VPL) thalamic nuclei, and their cortical projection is mainly preserved within the studied circuit.

Patients showed clear alterations and interhemispheric asymmetry of M30 latency, ECD strength, and position. The reduction of the M30 strength can reflect a decrease of local neuronal synchrony within the S1 inhibitory structures and a reduced M1 pyramidal neurons responsiveness to S1 projection. The unbalance between the S1 inhibitory and M1 excitatory activations is also indicated by the M30 ECD intra- and interhemispheric displace- ments, more pronounced in patients with higher EDSS (2 $<$ EDSS $<3.5$ ). The dysfunction of the S1 inhibitory network could extend previous finding of reduced GABA transmission in motor areas of MS patients [Dutta et al., 2006]. Our data suggest a reduction of M1 responsiveness to primary parietal cortical projections. Studies with transcranial magnetic stimulation (TMS) demonstrated that the resting motor threshold and the recruitment curve were within normative range in the remitting phase of MS, indicating normal motor cortical excitability [Caramia et al., 2004; Jorgensen et al., 2005]. Nevertheless, the M1 excitability to TMS, with a strong postsynaptic axonal activation, is not directly comparable to the M1 responsiveness to parietal projections. Data consistent with an altered sensory and motor communication come from prolonged TMS silent period [Caramia et al., 2004; Tataroglu et al., 2003] and delayed long-latency reflexes [Bonfiglio et al., 2006; Tataroglu et al., 2004] in MS patients, as well.

Morf_S1M1 in RR-MS patients was able to concisely sum up the bilateral spatial displacements of M30 generators, their strength reduction, and latency delays occurring in an uncorrelated manner in the two hemispheres. Morf_S1M1 shares the sensitivity of the standard N20 assessed by somatosensory evoked potentials (SEPs) in detecting a neural damage even in the absence of sensory symptoms. The advantage of Morf_S1M1, with respect to the standard SEP analysis, is that it allows the investigation of short-range intracortical connectivity, in terms of recruitment dynamics brought about by involved nodes projection properties.

A trend of thalamus volume reduction was documented in our MS patients, associated with lesion load [Ceccarelli et al., 2008].

Thalamic damage might be the consequence of local inflammatory activity [Brownell and Hughes, 1962]. Alternatively, its strong intracerebral connectivity might make the thalamus particularly susceptible to wallerian degeneration phenomena [Houtchens et al., 2007]. This latter hypothesis could be supported by the observation that thalamic volume reduction was found only in the left dominant hemisphere.

Previous data in MS patients linked the damage of the principal white-matter bundles involved in motor control to the alteration of movement-related functional connectivity among engaged areas [Rocca et al., 2007]. In our patients, S1 responsiveness was preserved in terms of timing, number, and position of synchronously recruited neurons (subtending M20 ECD, called here node 1 for convenience). The absence of a direct projection from thalamic VPL nuclei to M1 [Asanuma et al., 1979, 1980; Huffman and Krubitzer, 2001] implies that the successively recruited neuronal pool (subtending M30 ECD, node 2) receives direct projection mainly from node 1 . Three alternative not mutually exclusive hypotheses can be formulated about the origin of the node 2 activation distortion (see Fig. 6): 


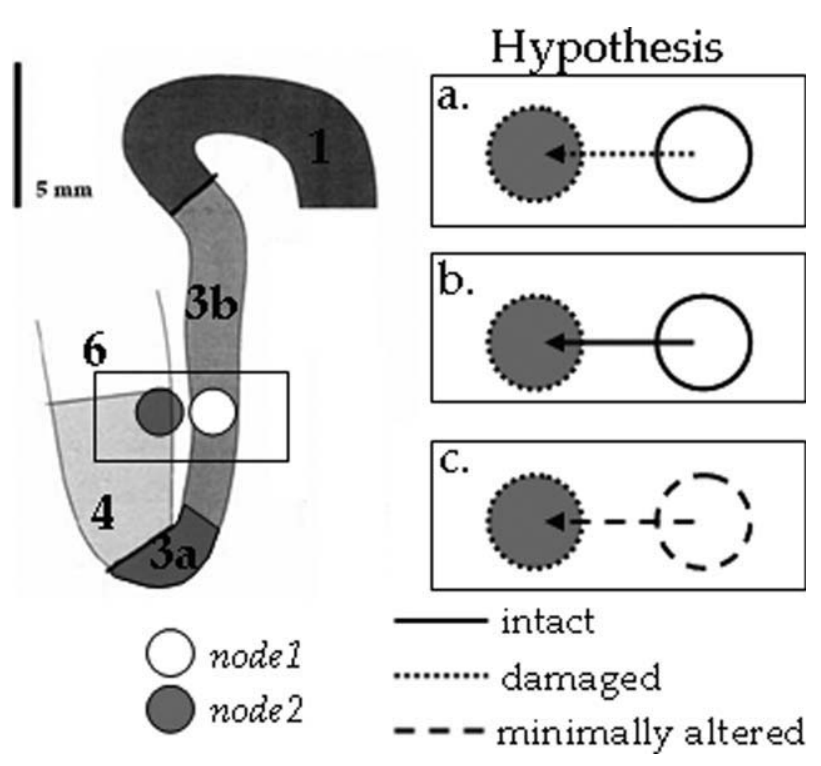

Figure 6.

Hypothesis behind M30 ECD distortions. See text for proposed hypothesis.

a. node 1 sends an altered input signal to node 2, indicating a functional intracortical disconnection in the S1-M1 area in spite of a preserved S1 excitability to thalamic projection;

b. node 1 sends a preserved input signal to node 2, which defectively activates, as an effect of its involvement in other damaged networks;

c. node 1 activates with minimal alterations-although within normal range-but its only distorted projection to node 2 gives rise to an amplified alteration.

Remarkable Morf_S1M1 reduction speaks in favor of hypothesis 1 . In favor to hypothesis 2 speaks the frequently coexistent involvement in MS of S1, M1, and thalamus, which are key stations of most multimodal integrative small- and long-range networks. In addition to that, leftsided prevalence of both thalamic volume reduction and its correlation with intracortical disarrangement would also support hypothesis 2, inferring a greater sensitivity to disconnection damage of nodes involved in frequently recruited and highly specialized neural networks [Amunts et al., 2003, 2007; Herve et al., 2005; Zappasodi et al., 2006].

Even in mildly disabled patients, MS profoundly impaired the SEF morphology, a sensitive individual anatomo-functional marker of primary somatosensory network patterning. An intracortical dynamic disconnection was demonstrated within the primary sensorimotor area, the main hub of most multimodal integrative small- and long-range networks. The impairment of intracortical information flow was found to be associated with the thalamic volume reduction in the dominant hemisphere. Our data support the hypothesis of MS as a disconnection syn- drome, with a greater susceptibility to damage revealed in nodes belonging to frequently recruited and highly specialized networks.

\section{ACKNOWLEDGMENTS}

The authors are grateful to Matilde Ercolani for irreplaceable technical support.

\section{REFERENCES}

Ahsan RL, Allom R, Gousias IS, Habib H, Turkheimer FE, Free S, Lemieux L, Myers R, Duncan JS, Brooks DJ, Koepp MJ, Hammers A (2007): Volumes, spatial extents and a probabilistic atlas of the human basal ganglia and thalamus. Neuroimage 38:261-270.

Amunts K, Schleicher A, Ditterich A, Zilles K (2003): Broca's region: Cytoarchitectonic asymmetry and developmental changes. J Comp Neurol 465:72-89.

Amunts K, Armstrong E, Malikovic A, Homke L, Mohlberg H, Schleicher A, Zilles K (2007): Gender-specific left-right asymmetries in human visual cortex. J Neurosci 27:1356-1364.

Asanuma H, Larsen KD, Yumiya H (1979): Receptive fields of thalamic neurons projecting to the motor cortex in the cat. Brain Res 172:217-228.

Asanuma H, Larsen K, Yumiya H (1980): Peripheral input pathways to the monkey motor cortex. Exp Brain Res 38:349-355.

Au Duong MV, Boulanouar K, Audoin B, Treseras S, Ibarrola D, Malikova I, Confort-Gouny S, Celsis P, Pelletier J, Cozzone PJ, Ranjeva JP (2005): Modulation of effective connectivity inside the working memory network in patients at the earliest stage of multiple sclerosis. Neuroimage 24:533-538.

Audoin B, Au Duong MV, Malikova I, Confort-Gouny S, Ibarrola D, Cozzone PJ, Pelletier J, Ranjeva JP (2006): Functional magnetic resonance imaging and cognition at the very early stage of MS. J Neurol Sci 245:87-91.

Audoin B, Guye M, Reuter F, Au Duong MV, Confort-Gouny S, Malikova I, Soulier E, Viout P, Cherif AA, Cozzone PJ, Pelletier J, Ranjeva JP (2007): Structure of WM bundles constituting the working memory system in early multiple sclerosis: A quantitative DTI tractography study. Neuroimage 36:13241330.

Barbati G, Porcaro C, Zappasodi F, Rossini PM, Tecchio F (2004): Optimization of an independent component analysis approach for artifact identification and removal in magnetoencephalographic signals. Clin Neurophysiol 115:1220-1232.

Bonfiglio L, Rossi B, Sartucci F (2006): Prolonged intracortical delay of long-latency reflexes: Electrophysiological evidence for a cortical dysfunction in multiple sclerosis. Brain Res Bull 69:606-613.

Brett M, Leff AP, Rorden C, Ashburner J (2001): Spatial normalization of brain images with focal lesions using cost function masking. Neuroimage 14:486-500.

Brownell B, Hughes JT (1962): The distribution of plaques in the cerebrum in multiple sclerosis. J Neurol Neurosurg Psychiatry 25:315-320.

Cader S, Cifelli A, Abu-Omar Y, Palace J, Matthews PM (2006): Reduced brain functional reserve and altered functional connectivity in patients with multiple sclerosis. Brain 129:527-537. 
Cader S, Johansen-Berg H, Wylezinska M, Palace J, Behrens TE Smith S, Matthews PM (2007): Discordant white matter N-acetylasparate and diffusion MRI measures suggest that chronic metabolic dysfunction contributes to axonal pathology in multiple sclerosis. Neuroimage 36:19-27.

Calabrese P (2006): Neuropsychology of multiple sclerosis-An overview. J Neurol 253 (Suppl 1): I10-I15.

Caramia MD, Palmieri MG, Desiato MT, Boffa L, Galizia P, Rossini PM, Centonze D, Bernardi G (2004): Brain excitability changes in the relapsing and remitting phases of multiple sclerosis: A study with transcranial magnetic stimulation. Clin Neurophysiol 115:956-965.

Casadio M, Sanguineti V, Morasso P, Solaro C (2008): Abnormal sensorimotor control, but intact force field adaptation, in multiple sclerosis subjects with no clinical disability. Mult Scler 14:330-342.

Ceccarelli A, Rocca MA, Pagani E, Colombo B, Martinelli V, Comi G, Filippi M (2008): A voxel-based morphometry study of grey matter loss in MS patients with different clinical phenotypes. Neuroimage 42:315-322.

Cercignani M, Bozzali M, Iannucci G, Comi G, Filippi M (2002): Intra-voxel and inter-voxel coherence in patients with multiple sclerosis assessed using diffusion tensor MRI. J Neurol 249:875-883.

Charcot J (1868): Histologie de la sclerose en plaques. Gazette des hopitaux. Paris 41: pp 554-555.

Cifelli A, Arridge M, Jezzard P, Esiri MM, Palace J, Matthews PM (2002): Thalamic neurodegeneration in multiple sclerosis. Ann Neurol 52:650-653.

Creutzfeldt OD (1977): Generality of the functional structure of the neocortex. Naturwissenschaften 64:507-517.

De Luca M, Smith S, De Stefano N, Federico A, Matthews PM (2005): Blood oxygenation level dependent contrast resting state networks are relevant to functional activity in the neocortical sensorimotor system. Exp Brain Res 167:587-594.

Del Gratta C, Pizzella V, Tecchio F, Romani GL (2001): Magnetoencephalography-A noninvasive brain imaging method with 1 ms time resolution. Rep Prog Phys 64:1759-1814.

Desmedt JE, Ozaki I (1991): SEPs to finger joint input lack the N20-P20 response that is evoked by tactile inputs: Contrast between cortical generators in areas $3 \mathrm{~b}$ and 2 in humans. Electroencephalogr Clin Neurophysiol 80:513-521.

Dutta R, McDonough J, Yin X, Peterson J, Chang A, Torres T, Gudz T, Macklin WB, Lewis DA, Fox RJ, Rudick R, Mirnics K, Trapp BD (2006): Mitochondrial dysfunction as a cause of axonal degeneration in multiple sclerosis patients. Ann Neurol 59:478-489.

Duvernoy HM (1991): The Human Brain: Surface, Three-Dimensional Sectional Anatomy and MRI. New York: SpringerVerlag.

Fabiano AJ, Sharma J, Weinstock-Guttman B, Munschauer FE III, Benedict RH, Zivadinov R, Bakshi R (2003): Thalamic involvement in multiple sclerosis: A diffusion-weighted magnetic resonance imaging study. J Neuroimaging 13:307-314.

Hari R, Reinikainen K, Kaukoranta E, Hamalainen M, Ilmoniemi R, Penttinen A, Salminen J, Teszner D (1984): Somatosensory evoked cerebral magnetic fields from SI and SII in man. Electroencephalogr Clin Neurophysiol 57:254-263.

He Y, Dagher A, Chen Z, Charil A, Zijdenbos A, Worsley K, Evans A: Impaired small-world efficiency in structural cortical networks in multiple sclerosis associated with white matter lesion load. Brain 2009 (in press).
Herve PY, Mazoyer B, Crivello F, Perchey G, Tzourio-Mazoyer N (2005): Finger tapping, handedness and grey matter amount in the Rolando's genu area. Neuroimage 25:1133-1145.

Horwitz B (2003): The elusive concept of brain connectivity. Neuroimage 19:466-470.

Houtchens MK, Benedict RH, Killiany R, Sharma J, Jaisani Z, Singh B, Weinstock-Guttman B, Guttmann CR, Bakshi R (2007): Thalamic atrophy and cognition in multiple sclerosis. Neurology 69:1213-1223.

Huang MX, Aine C, Davis L, Butman J, Christner R, Weisend M, Stephen J, Meyer J, Silveri J, Herman M, Lee RR (2000): Sources on the anterior and posterior banks of the central sulcus identified from magnetic somatosensory evoked responses using multistart spatio-temporal localization. Hum Brain Mapp 11:59-76.

Huffman KJ, Krubitzer L (2001): Area 3a: Topographic organization and cortical connections in marmoset monkeys. Cereb Cortex 11:849-867.

Ilmoniemi RJ (1993): Models of source currents in the brain. Brain Topogr 5:331-336.

Inglese M, Ge Y, Filippi M, Falini A, Grossman RI, Gonen O (2004): Indirect evidence for early widespread gray matter involvement in Relapsing-Remitting multiple sclerosis. Neuroimage 21:1825-1829.

Inglese M, Park SJ, Johnson G, Babb JS, Miles L, Jaggi H, Herbert J, Grossman RI (2007): Deep gray matter perfusion in multiple sclerosis: Dynamic susceptibility contrast perfusion magnetic resonance imaging at $3 \mathrm{~T}$. Arch Neurol 64:196-202.

Jorgensen LM, Nielsen JE, Ravnborg M (2005): MEP recruitment curves in multiple sclerosis and hereditary spastic paraplegia. J Neurol Sci 237:25-29.

Kawamura T, Nakasato N, Seki K, Kanno A, Fujita S, Fujiwara S, Yoshimoto T (1996): Neuromagnetic evidence of pre- and postcentral cortical sources of somatosensory evoked responses. Electroencephalogr Clin Neurophysiol 100:44-50.

Kurtzke JF (1983): Rating neurologic impairment in multiple sclerosis: An expanded disability status scale (EDSS). Neurology 33:1444-1452.

Leocani L, Comi G (1999): EEG coherence in pathological conditions. J Clin Neurophysiol 16:548-555.

Lowe MJ, Phillips MD, Lurito JT, Mattson D, Dzemidzic M, Mathews VP (2002): Multiple sclerosis: Low-frequency temporal blood oxygen level-dependent fluctuations indicate reduced functional connectivity initial results. Radiology 224:184-192.

Lublin FD, Reingold SC (1996): Defining the clinical course of multiple sclerosis: Results of an international survey. National Multiple Sclerosis Society (USA) Advisory Committee on Clinical Trials of New Agents in Multiple Sclerosis. Neurology 46:907-911.

Mauguiere F, Merlet I, Forss N, Vanni S, Jousmaki V, Adeleine P, Hari R (1997): Activation of a distributed somatosensory cortical network in the human brain: A dipole modelling study of magnetic fields evoked by median nerve stimulation, Part 2: Effects of stimulus rate, attention and stimulus detection. Electroencephalogr Clin Neurophysiol 104:290-295.

Mesaros S, Rocca MA, Absinta M, Ghezzi A, Milani N, Moiola L, Veggiotti P, Comi G, Filippi M (2008): Evidence of thalamic gray matter loss in pediatric multiple sclerosis. Neurology 70:1107-1112.

Mima T, Terada K, Maekawa M, Nagamine T, Ikeda A, Shibasaki H (1996): Somatosensory evoked potentials following proprioceptive stimulation of finger in man. Exp Brain Res 111:233245. 
Niimi M, Ohira T, Akiyama T, Hiraga K, Kaneko Y, Ochiai M, Fukunaga A, Kobayashi M, Kawase T (2008): Source analysis of the magnetic field evoked during self-paced finger movements. Neurol Res 30:239-243.

Oldfield RC (1971): The assessment and analysis of handedness: The Edinburgh inventory. Neuropsychologia 9:97-113.

Ormerod IE, Miller DH, McDonald WI, du Boulay EP, Rudge P, Kendall BE, Moseley IF, Johnson G, Tofts PS, Halliday AM, Bronstein AM, Scaravilli F, Harding AE, Barnes D, Zilkha KJ. (1987): The role of NMR imaging in the assessment of multiple sclerosis and isolated neurological lesions. A quantitative study. Brain 110 (Pt 6):1579-1616.

Romani GL, Pizzella V (1990): Localization of brain activity with magnetoencephalography. Adv Neurol 54:67-77.

Reddy H, Narayanan S, Arnoutelis R, Jenkinson M, Antel J, Matthews PM, Arnold DL (2000): Evidence for adaptive functional changes in the cerebral cortex with axonal injury from multiple sclerosis. Brain 123 (Pt 11):2314-2320.

Reich DS, Smith SA, Zackowski KM, Gordon-Lipkin EM, Jones CK, Farrell JA, Mori S, van Zijl PC, Calabresi PA (2007): Multiparametric magnetic resonance imaging analysis of the corticospinal tract in multiple sclerosis. Neuroimage 38:271-279.

Restuccia D, Valeriani M, Barba C, Le Pera D, Tonali P, Mauguiere F (1999): Different contribution of joint and cutaneous inputs to early scalp somatosensory evoked potentials. Muscle Nerve 22:910-919.

Rocca MA, Pagani E, Absinta M, Valsasina P, Falini A, Scotti G, Comi G, Filippi M (2007): Altered functional and structural connectivities in patients with MS: A 3-T study. Neurology 69:2136-2145.

Rocca MA, Absinta M, Ghezzi A, Moiola L, Comi G, Filippi M (2008): Is a preserved functional reserve a mechanism limiting clinical impairment in pediatric MS patients? Hum Brain Mapp 30:2844-2851.

Rossini PM, Barker AT, Berardelli A, Caramia MD, Caruso G, Cracco RQ, Dimitrijevic MR, Hallett M, Katayama Y, Lucking $\mathrm{CH}$, Maertens de Noordhout AL, Marsden CD, Murray NMF, Rothwell JC, Swash M, Tomberg C (1994): Non-invasive electrical and magnetic stimulation of the brain, spinal cord and roots: Basic principles and procedures for routine clinical application. Report of an IFCN committee. Electroencephalogr Clin Neurophysiol 91:79-92.

Schmahmann JD, Pandya DN (2008): Disconnection syndromes of basal ganglia, thalamus, and cerebrocerebellar systems. Cortex 44:1037-1066.

Sepulcre J, Sastre-Garriga J, Cercignani M, Ingle GT, Miller DH, Thompson AJ (2006): Regional gray matter atrophy in early primary progressive multiple sclerosis: A voxel-based morphometry study. Arch Neurol 63:1175-1180.

Tataroglu C, Genc A, Idiman E, Cakmur R, Idiman F (2003): Cortical silent period and motor evoked potentials in patients with multiple sclerosis. Clin Neurol Neurosurg 105:105-110.

Tataroglu C, Genc A, Idiman E, Cakmur R, Idiman F (2004): Cortical relay time for long latency reflexes in patients with definite multiple sclerosis. Can J Neurol Sci 31:229-234.

Tecchio F, Rossini PM, Pizzella V, Cassetta E, Romani GL (1997): Spatial properties and interhemispheric differences of the sensory hand cortical representation: A neuromagnetic study. Brain Res 767:100-108.

Tecchio F, Pasqualetti P, Pizzella V, Romani G, Rossini PM (2000): Morphology of somatosensory evoked fields: Inter-hemispheric similarity as a parameter for physiological and pathological neural connectivity. Neurosci Lett 287:203-206.

Tecchio F, Zappasodi F, Pasqualetti P, Rossini PM (2005): Neural connectivity in hand sensorimotor brain areas: An evaluation by evoked field morphology. Hum Brain Mapp 24:99-108.

Valeriani M, Restuccia D, Di Lazzaro V, Le Pera D, Barba C, Tonali P, Mauguiere F (1998): Dipolar sources of the early scalp somatosensory evoked potentials to upper limb stimulation. Effect of increasing stimulus rates. Exp Brain Res 120:306-315.

Wylezinska M, Cifelli A, Jezzard P, Palace J, Alecci M, Matthews PM (2003): Thalamic neurodegeneration in relapsing-remitting multiple sclerosis. Neurology 60:1949-1954.

Zappasodi F, Pasqualetti P, Tombini M, Ercolani M, Pizzella V, Rossini PM, Tecchio F (2006): Hand cortical representation at rest and during activation: Gender and age effects in the two hemispheres. Clin Neurophysiol 117:1518-1528. 